\title{
Categorization of Narrative Semantics for Use in Generative Multidocument Summarization
}

\author{
INLG04-1319438223
}

No Institute Given

\begin{abstract}
The generative summarization of textual stories has been one of the goals of computational narratology since attempts at full semantic NLU in the '70s. Our NLP group has recently created several systems for multidocument news summarization using purely statistical methods. Between these poles, there may be an unexplored avenue where knowledge of story structure can give partial, yet useful semantic understanding to a news reader. Such knowledge can then lead to summaries more informed than those based on solely statistical means. This student paper represents work in progress on a two-module system: The first module categorizes news articles into their underlying dramatic structures; the second will attempt to use this understanding to create and execute a generative plan, concisely retelling the story to form a surface-level summary.
\end{abstract}

\section{Introduction}

Content selection is a limiting factor in many generation systems, especially those intended for summarization. Statistical summarizers that rely purely on sentence extraction, while practical on unrestricted texts, often produce summaries too "close" to the sources. Without any semantic insight into the gist of the text, a statistical summarizer can favor sentences with nonessential details over those that tell salient events.

While full semantic understanding of an arbitrary text is not a practical goal, the domain of news summarization imposes a restriction on many its texts that one may be able to exploit: news is, at its core, an act of storytelling. If a news event does not arouse fear, or compassion, or a laugh, or some other emotional response to the dramatic underpinnings of the story, it does not make the pages of a broadsheet.

In this paper, we describe the initial stages of a project to create a new model of the rhetorical structure of textual stories and apply it to the content selection piece of a generative multidocument news summarizer.

\section{Related Work}

The link between news and storytelling has been investigated before, originally by those attempting full semantic understanding in the '70s [17]. More recently, 
researchers such as Power [13], Mann and Thompson [9] have developed rhetorical structure theory as a more practical, high-level representation of a general text's structure. Plans have been used by Hovy [7], Moore and Paris [12] to improve text generation for expert systems, as have schemas by McKeown [11].

In parallel with this work, computational narratology has aimed to capture the essence of drama for both story understanding and generation. The different approaches vary widely: Some place the notion of conflict between characters at the atomic center of their models [18]; others prefer surface-level syntactic structure [16], the autonomy of self-determining characters [15], the human process of storytelling [8] or even the reader's emotional response to a story [2].

Computational narratology has experienced a revival in recent years, by both theorists and those bringing practical applications to bear. The introductory paper by Mateas and Sengers [10] from the 1999 AAAI Fall Symposium gives an excellent overview, as does the summary of the MIT Media Lab's reading group on narrative intelligence by Davis and Travers [4]. However, little exploration has been done of the potential for a fusion of narratology and rhetorical structure theory for summarization.

\section{System Design}

Telling interesting narratives is a skill that journalists and writers of fiction alike are trained to do. They each look for the best "angle" with which to cast the events of their worlds into a dramatic mold.

One of the motifs in the heritage of literary theory, where computational limitations are not even considered, has been the idea that there are only so many stories that can be told ([1], [14]). Within the news domain, there are even fewer, as journalists strive to sell their stories to the public by invoking plots, characters, and themes that have been shown to generate interest in the past (e.g., the death of the unsuspecting innocent; the fall from power; the personal attack; the rich magnate; the comeback or recovery; justice for the evil; new technology for old wants).

Each of these plots, characters and themes are metadata for a story that carry certain narrative connotations. For example, a fall from power article probably describes the person in question, portrays the person as a villain brought to justice or a hero wrongfully hindered, exposits the reason for the fall, and characterizes the opposing force. As these are the essentials of the fall from power story, they represent the facts most important to the reader, and as such, they are the best facts to portray in a summary. If, by contrast, an article tells of the death of the unsuspecting innocent, the corresponding content selection problem is slightly different.

In other words, by categorizing an article into one or several of a reasonably small number of plot elements, we can invoke a generative plan that is customized for retelling that particular story. Such a system would combine the knowledgebased and the statistical: each of the possible stories would be coded a priori with its structural properties, but the content planner and surface-level generator 
would be statistical, using extant tools such as FUF/SURGE [6]. This approach would create summaries more informed than those by pure sentence extraction, while avoiding the rigidness of complete semantic understanding and working for real texts in an online environment.

Our system will execute such a process by using two modules: a story categorizer and a plan generator.

\subsection{Story Categorizer}

The categorizer, partially implemented, determines the narrative backbone of a story, choosing the plot elements that most closely apply to the text. There are several intersecting categorization problems that lie at this stage.

For one, not all news articles are stories in the straightforward sense, declaratively describing events unknown to the reader. The categorizer uses syntactic and lexical features to distinguish between several structural patterns of news articles:

1. Articles that tell a highly narrative story ("hard news")

2. Articles that are more digressive than "hard news," such as:

- Articles that provide an analysis, historical perspective, or opinion/editorial

- Character studies or articles that identify an emerging trend

Once the categorizer can isolate the highly narrative portions of a set of documents, it will more accurately be able to make further distinctions to determine the participating plot elements, such as:

- Is the protagonist losing something, gaining something, fighting to reclaim something lost, or fighting to gain something new?

- Does that goal take the form of romance, freedom, privacy, peace, security, power, recuperation or survival?

- Does the protagonist face competition from a rival, an enemy, an authority, nature or limitations of a social class?

We are continuing to develop our narrative model (i.e., the story elements available to the categorizer), and plan on utilizing statistical categorization techniques over large training sets to achieve this step. There may, for example, be a particular set of words or syntactic relationships that identify a redemption from past mistakes story, or one about family bonding.

Our current progress in implementing the categorizer is described below.

\subsection{Plan Generator}

Once the narrative elements of a news article are identified, content selection and regeneration will be able to capitalize upon the limited semantic knowledge.

Each story element will have an associated set of "arguments": names of people, places, and other details that concretize the abstract narrative pieces. These 
arguments will be essential for the generative piece of the summarizer; working from the output of a high-accuracy statistical parser [3], the categorizer will also perform knowledge acquisition as it goes. Other statistical content selection will be performed in methods similar to those in related studies [5]. The plan generator will then perform sentence planning, also in ways that are specific to the associated story elements. For instance, the "root" event might be explicated first, followed by details and complications.

Finally, the generative plan will be executed by a surface-level lexical chooser [6], which will produce a final summary.

\section{Initial Results}

As we have mentioned, the initial step in this project has been the development of a model of common plots, characters, and themes of which most news articles are instances. Several examples have already been given, such as the fall from power. The challenge at this stage is to identify elements that are general enough to be complete and enumerable, yet specific enough to be useful. For example, almost all news can fall into the plot a character wants something but has difficulty in getting it; such a model would be complete, but too vague to be useful. Yet once plots get too specific, they become too knowledge-dependent, and the data are too sparse to train for them.

Simultaneously, we have implemented a prototype for the first task of the categorizer, as described above. Working from a statistical parse, it uses syntactic rules to decide whether a sentence is one of declarative, highly narrative "hard news," or a more digressive detail. (We don't yet distinguish between the subtypes of digressive sentences.) The features include:

- Whether a sentence is attributed, or is a direct quotation of some source

- Whether there is a clear subject-action pattern

- Whether the subject is in the first or third person

- Whether the subject is a noun phrase or a pronoun

We are testing the categorizer on a collection of over 350 first sentences of articles, which are manually labeled as either "narrative" or "digressive." (We hypothesize that the first sentence of an article will be the richest source for key words and phrases for story categorization, as it is good form for journalists to summarize the narrative essence of their stories in their leads.) Initial results indicate that the categorizer achieves $78 \%$ precision and $78 \%$ recall for the task of identifying narrative sentences from digressive ones.

\section{Conclusion}

In this paper, we have described a project in an intermediate stage that seeks to use high-level narrative semantics as a means for generating news summaries more informed than those that operate on purely statistical means. We have 
identified several of the plot elements we plan to encode, and implemented a prototype for the categorizer that distinguishes highly narrative sentences from more digressive ones. In the near future, we will continue to implement the categorizer, and the plan generator to follow.

\section{References}

1. Aristotle. Poetics. Available at http://www.gutenberg.net/etext99/poetc10.txt.

2. P. Bailey. Searching for storiness: Story-generation from a reader's perspective. In Mateas and Sengers [10].

3. M. Collins. A new statistical parser based on bigram lexical dependencies. In Arivind Joshi and Martha Palmer, editors, Proceedings of the Thirty-Fourth Annual Meeting of the Association for Computational Linguistics, pages 184-191, San Francisco, 1996. Morgan Kaufmann Publishers.

4. M. Davis and M. Travers. A brief overview of the narrative intelligence reading group.

5. P. Duboue and K. McKeown. Statistical acquisition of content selection rules for natural language generation. In 2003 Conference on Empirical Methods for Natural Language Processing (EMNLP 2003), Sapporo, Japan, July 2003.

6. M. Elhadad and J. Robin. An overview of surge: A reusable comprehensive syntactic realization component, 1996.

7. E. Hovy. Automated discourse generation using discourse structure relations. Artificial Intelligence, 1993.

8. M. Lebowitz. Story telling and generalization. Proceedings of the Seventh Annual Conference of the Cognitive Science Society, (Berkeley, California), pp. 100-109, 1985, 1995.

9. W.C. Mann and S.A. Thompson. Rhetorical structure theory: Toward a functional theory of text organization. Text, 8(3):243 - 281.

10. M. Mateas and P. Sengers, editors. Narrative Intelligence. Carnegie Mellon University / Media Arts Research Studies, Sankt Augustin, Germany; Cornell University, 1999, bound 2003.

11. K. McKeown. Text Generation: Using Discourse Strategies and Focus Constratins to Generate Natural Langauge Text. Cambridge University Press, 1985.

12. J. D. Moore and C. L. Paris. Planning text for advisory dialogues: Capturing intentional and rhetorical information. Computational Linguistics, 19(4):651-695, 1993.

13. R. Power. Planning texts by constraint satisfaction. In Proceedings of the 18th International Conference on Computational Linguistics (COLING-2000), Saarbruecken, Germany, pages 642-648, 2000.

14. V. Propp. Morphology of the Folktale. Trans. Laurence Scott. Ed. Louis A. Wagner. 2nd edition. Univ of Texas Press, 1969.

15. M. Riedl. Actor conference: Character-focused narrative planning. In Mateas and Sengers [10].

16. D. Rumelhart. Notes on a schema for stories. In Representation and Understanding: Studies in Cognitive Science, Bobrow, D.G. and Collins A., eds, pages 211-236. New York: Academic Press, Inc., 1975.

17. R. Schank and C. Riesbeck, editors. Inside Computer Understanding: Five Programs Plus Miniatures. Hillsdale, New Jersey: Lawrence Erlbaum Associates, 1981.

18. N. Szilas. Interactive drama on computer: beyond linear narrative. In Mateas and Sengers [10]. 